\title{
RESPONSES OF SOME CANOLA VARIETIES (Brassica napus L.) TO NITROGEN FERTILIZER LEVELS UNDER DIFFERENT SOWING DATES IN A NEW RECLAIMED SOIL
}

(Received: 16. 10. 2011)

\author{
By \\ M. M. Asfour \\ Environmental Studies and Research Institute, Minufiya University
}

\begin{abstract}
The present study was carried out to investigate the influence of sowing dates and nitrogen fertilizer levels on yield of five canola varieties in a new reclaimed soil. The experiment took place at the experimental farm of the Environmental Studies and Research Institute, Minufiya University, Sadat City, Egypt, during 2007/2008 and 2008/2009 seasons. Complete block design with spilt split plot with three replications was used. Where the main plots included four sowing dates (15 October, first November, 16 November, first December). The sub -plots contained three nitrogen fertilizer levels $(30,60 \& 90 \mathrm{Kg}$ $\mathrm{N} /$ fed). The sub - sub plots were assigned to five canola varieties (Sedo, Duplo, Serw - 4, Pectol, Drakkar). The results indicated that, the first of November was the best sowing date, it gave the highest values of all the studied traits and the highest seed yield per plant (43.76 and $48.94 \mathrm{~g}$ )in both seasons. Best nitrogen fertilizer level was $90 \mathrm{Kg} \mathrm{N} /$ fed. It scored the highest mean values (45.20 and 46.92g)of seed yield per plant. Serw-4 gave the highest seed yield (64.63 and 71.56g) in both seasons, respectively. Serw-4 had the best response for nitrogen fertilizer especially when planted at $1^{\text {st }}$ November and gave the highest seed yield(87.97 and $99.98 \mathrm{~g} /$ plant) followed by Duplo variety $(75.57,93.90 \mathrm{~g}) /$ plant in both seasons, respectively. Sowing date at the first of November with $90 \mathrm{Kg} \mathrm{N}$ per fed. were recommended for maximizing seed yield in new reclaimed soils with poor content of available nitrogen. Serw -4 and Duplo were the best varieties under the conditions of this study.
\end{abstract}

Key words: Brassica napus, canola oil, nitrogen fertilizer, sowing dates.

\section{INTRODUCTION}

Canola oil is considered one of the most important oil crops allover the world, ranking the third largest source of edible oil following soybean and palm oil ( Francois, 1994). In Egypt, canola is a recent introduction, but has a bright future and hopefully to contribute in reducing edible oil deficiency, where the degree of self sufficient ratio in edible plant oils declined from $25.6 \%$ in 1980 to $12.3 \%$ in 1996 ( El-Tantawy and Soliman 1999).Growing canola in Egypt may become easier if it is grown in the less fertile and / or salt affected soils. Growing a crop in such soils may become successful if it could produce relatively high economic yield with low level of inputs mainly nitrogen fertilizer. Fertilization is an important vital factor affecting plant growth and the produced canola seed yield especially nitrogen fertilizer. Nitrogen is referred as a balance wheel of plant nutrition. Canola is extremely sensitive to nitrogen fertilizer, especially in the less fertile and / or salt affected soils. Also, nitrogen is one of the most important factors as a nutrient, which comprised about $50 \%$ of the dry matter of protoplasm in canola cells and is one of the essential elements for proper growth and development of the plant (Sing, 1984). Increasing nitrogen fertilizer levels significantly increased seed yield and yield components. (Chauhan et al.,1993 ; Sarandon et al.,1993; Hassan and El Hakeem, 1996; Saini and sidhu, 1997; Patel , 1998; Said and Keshta 1999; Sharief and Keshta 2000 and 2002 ; Leilah et al., 2003 \&Asfour, 2006).

Sowing date is an important factor that determines the length of growing season as well as yield. Early sowing of canola delayed flowering and reduced reflection of radiation during flowering which were important factors leading to the highest yield achieved by late sowing. Jenkins and Leitch, 1986; Jasinska et al., 1989 reported that seed yield /ha decreased with delay in sowing dates. Moreover, Leto et al., 1995 found that the optimum sowing date on mid - November produced the highest seed yield / ha, compared to sowing on 31 October, 30November or mid 
December. Starner et al., 1996 reported that the optimum sowing dates for rape cv. Cascade was at late September or early October .Miralles et al., 2001; stated that developmental patterns of canola were greatly affected by sowing dates. Sharief and Keshta (2002) stated that the best sowing dates for plant height, number of branches / plant as well as seed yield/plant were in the $5{ }^{\text {th }}$ November. In Egypt late sowing dates of canola causes extensive damage by Brevicoryne brassica $\mathrm{L}$, and serious losses of siliqua and seed yield . (Amany, 2009).

Therefore, this investigation is aimed to study the performance of some canola varieties under different nitrogen fertilizer levels and different sowing dates as well as their interaction in a new reclaimed soil as affected by salinity.

\section{MATERIALS AND METHODES}

The investigation was carried out during two winter successive seasons of 2007/2008 and 2008/2009 at the farm of the Environmental Studies and Research Institute, Minufiya University, Sadat city, to evaluate five of canola varieties under different levels of nitrogen fertilizer and different sowing dates. A split split plot design with three replications was used . The main plots included four sowing dates (15 October, First November, 16 November, \& first December). The sub Plots contained three nitrogen fertilizer levels $(30,60 \& 90 \mathrm{Kg} \mathrm{N} / \mathrm{fed}$. ). The sub - sub plots were assigned to five canola varieties (Sedo, Duplo, Serw - 4, Pactol, Drakkar ). The experimental unit included five ridges $70 \mathrm{~cm}$ apart and $3.5 \mathrm{~m}$ long contributing a plot area of 12.25 $\mathrm{m}^{2}$. The experimental soil was sandy loamy soil. The physical and chemical analysis of the experimental soil are shown in Table (1) The chemical analysis of the experimental soil was achieved according to Cottenie et al. (1982).

Nitrogen was applied in the form of ammonium nitrate $(33.5 \% \mathrm{~N})$ as three portions, the first was $20 \%$ added at sowing date as reactive portion, the second portion was $40 \%$ added after 30 days from sowing and the third was $40 \%$ added at the flowering stage. Phosphorus as calcium super- phosphate $\left(15.5 \% \mathrm{P}_{2} \mathrm{O}_{5}\right)$, at a rate of 150
$\mathrm{Kg} / \mathrm{fed}$ and potassium as potassium sulphate $\left(48 \% \mathrm{~K}_{2} \mathrm{O}\right)$ at a rate of $50 \mathrm{Kg} /$ fed were applied during soil preparation. Canola seeds were hand sown on one side of the ridge as the usual dry method . Plants were thinned to one plant per hill, $25 \mathrm{~cm}$ between hills to insure 24,000 plants per Fadden. The number of days to $50 \%$ flowering, was calculated from sowing dates for each sub-sub plot. Also, plant height $(\mathrm{cm})$, number of primary branches, number of siliqua per plant, number of seeds per siliqua, 1000 seeds weight, seed yield per plant and seed yield per Fadden were recorded at harvest on ten guarded plants taken at random from each sub-sub plot. Data of the two seasons were subjected to the proper statistical analysis as the technique of analysis of variance of split split plot design as mentioned by Gomez and Gomez (1984). Treatment mean values were compared using least significant difference (L.S.D) tested at 0.05 level of probability.

\section{RESULTS AND DISCUSSION}

The analysis of variance in Table 2 indicated that there were high significant differences among all sowing dates in all studied traits in 2007/2008 and 2008/2009 season. This may be due to the variation in weather condition such as the length of light period for all sowing dates under study. Also, data showed that the nitrogen fertilizer levels had significantly effect on all studied traits in both season. Interaction between sowing dates and nitrogen fertilizer levels had significant effect in all the studied traits. Also, there were high significant differences among all varieties in all the studied traits in 2007/2008 and 2008/2009 seasons. This may be due to genetic background differences among all varieties under study. Interaction between sowing dates and varieties showed high significant effect on all the studied traits in both years. Interaction between nitrogen fertilizer levels and varieties showed high significant effect on all the studied traits in both seasons. Interaction among sowing dates, nitrogen fertilizer levels and varieties showed high significant effect on all the studied traits in both seasons.

Table (1): Some physical and chemical properties of the experimental field soil in the two seasons.

\begin{tabular}{|c|c|c|c|c|c|c|c|c|c|}
\hline \multirow{2}{*}{ Season } & \multicolumn{2}{|c|}{$\begin{array}{c}\text { Chemical } \\
\text { properties }\end{array}$} & \multicolumn{3}{|c|}{ Available (mg/kg) } & \multicolumn{5}{c|}{ Physical properties } \\
\cline { 2 - 10 } & pH & Ec ds/m & N & P & K & Sand & Silt & Clay & Texture \\
\hline $\mathbf{2 0 0 7 / 2 0 0 8}$ & 7.41 & 1.78 & 11.80 & 5.86 & 32.4 & 72.78 & 19.34 & 7.88 & Sand loamy \\
\hline $\mathbf{2 0 0 8 / 2 0 0 9}$ & 7.42 & 1.80 & 12.00 & 6.07 & 33.2 & 72.74 & 19.37 & 7.89 & Sand loamy \\
\hline
\end{tabular}


Table ( 2 ) : Mean squares of ordinary analysis of sowing date, nitrogen fertilizer levels, and varieties and their interaction for all the studied traits in $(2007 / 2008)$. and $(2008 / 2009)$ season.

\begin{tabular}{|c|c|c|c|c|c|c|c|c|}
\hline S.O.V & \begin{tabular}{|c|}
$\begin{array}{c}\text { Days to 50\% } \\
\text { flowering }\end{array}$ \\
\end{tabular} & $\begin{array}{l}\text { Plant height } \\
\text { (cm) }\end{array}$ & $\begin{array}{c}\text { No . of primary } \\
\text { branches / Plant }\end{array}$ & $\begin{array}{c}\text { No . of siliquas } \\
\text { / plant }\end{array}$ & $\begin{array}{c}\text { No. of seeds } \\
\text { /siliqua }\end{array}$ & $\begin{array}{c}1000 \text { seed } \\
\text { weight }(\mathrm{g})\end{array}$ & $\begin{array}{l}\text { Seed yield / } \\
\text { plant ( g) }\end{array}$ & $\begin{array}{c}\text { Seed yield/ Fed } \\
(\mathrm{kg})\end{array}$ \\
\hline \multicolumn{9}{|c|}{$2007 / 2008$ season } \\
\hline Sowing Date(SD) & $29.71^{* *}$ & $136.884^{* * *}$ & 54.979* $^{* *}$ & $109399.338^{* *}$ & $6.694^{* * *}$ & $0.283^{* *}$ & 841.856 "** & 483902.382 \\
\hline Error (a) & 0.828 & 2.462 & 0.299 & 783.972 & 0.026 & 0.004 & 6.780 & 3700.684 \\
\hline Nitrogen $(\mathrm{N})$ & $94.21^{* *}$ & $1162.685^{* *}$ & $101.938^{* * *}$ & 503215.382 $^{* *}$ & $6.218^{* *}$ & $0.265^{* *}$ & $3495.38^{* * *}$ & $2017147.47^{* *}$ \\
\hline $\mathbf{S D} \times \mathbf{N}$ & $12.54^{* *}$ & $104.63^{* *}$ & $1.428^{*}$ & $26112.755^{* * *}$ & $3.394^{* *}$ & $\mathbf{0 . 1 1 1}^{* *}$ & $265.727^{* *}$ & $156011.175^{* *}$ \\
\hline Error (b) & 0.71 & 2.848 & 0.536 & 678.164 & 0.022 & 0.006 & 4.143 & 2546.960 \\
\hline Varieties (V) & $569.34^{* *}$ & $1412.83^{* *}$ & $137.644^{* *}$ & $392777.69{ }^{* *}$ & $56.056^{* *}$ & $5.818^{* *}$ & $8732.002^{* *}$ & $5033623.112^{* * *}$ \\
\hline $\mathbf{S D} \times \mathbf{V}$ & $2.61^{* *}$ & $21.879^{* *}$ & $2.537^{* *}$ & $1662.189^{*}$ & $1.123^{* *}$ & $0.042^{* *}$ & $43.107^{* *}$ & $24731.047^{* *}$ \\
\hline $\mathbf{N} \times \mathbf{V}$ & $4.14^{* * \pi}$ & 50.379 $^{2 * F}$ & $1.933^{* * *}$ & $2858.272^{* * *}$ & $1.446^{* * 6}$ & $0.046^{* * *}$ & $123.029^{* * *}$ & $71346.758^{* *}$ \\
\hline $\mathbf{S D} \times \mathbf{N} \times \mathbf{V}$ & $5.07^{* *}$ & 31.11 $^{* *}$ & $2.382^{* *}$ & $6189.746^{* *}$ & $1.872^{* *}$ & $0.064^{* *}$ & $48.462^{* * *}$ & $27986.485^{* *}$ \\
\hline Error $(C)$ & 0.576 & 2.939 & 0.363 & 791.889 & 0.033 & 0.008 & 5.166 & 3058.896 \\
\hline \multicolumn{9}{|c|}{$2008 / 2009$ season } \\
\hline Sowing Date(SD) & $34.954^{* *}$ & $432.216^{* *}$ & $27.602^{* *}$ & $280981.178^{* *}$ & $12.723^{* *}$ & $0.705^{* *}$ & $2947.037^{* *}$ & $1680232.4233^{* *}$ \\
\hline Error (a) & 0.143 & 1.408 & 0.418 & 1119.137 & 0.05 & 0.006 & 11.777 & 10103.944 \\
\hline Nitrogen $(\mathbf{N})$ & $111.872^{* *}$ & $562.045^{* *}$ & $57.5666^{* *}$ & $301812.82^{* *}$ & $12.152^{* *}$ & $3.272^{* *}$ & $4437.873^{* *}$ & $2645108.095^{* *}$ \\
\hline $\mathbf{S D} \times \mathbf{N}$ & $5.198^{* *}$ & $96.498^{* *}$ & $7.063^{* *}$ & $5718.567^{* *}$ & $1.719^{* *}$ & $0.042^{* *}$ & $93.005^{* *}$ & $55560.5522^{* *}$ \\
\hline Error (b) & 0.117 & 6.604 & 0.124 & 533.481 & 0.051 & 0.010 & 6.156 & 4062.437 \\
\hline Varieties (V) & $674.250^{* *}$ & $1319.264^{* *}$ & $107.486{ }^{* *}$ & $705499.44^{* * *}$ & $61.482^{* *}$ & $5.462^{* *}$ & $12741.684^{* *}$ & $7391745.64^{* *}$ \\
\hline $\mathbf{S D} \times \mathbf{V}$ & $3.787^{* *}$ & $27.277^{* *}$ & $0.967^{* * *}$ & $12480.14^{* *}$ & $1.455^{* *}$ & $0.025^{*}$ & $188.376{ }^{* *}$ & $109658.895^{* *}$ \\
\hline $\mathbf{N} \times \mathbf{V}$ & $0.796^{* *}$ & $29.965^{* *}$ & $0.571^{* * *}$ & $4438.21^{* * *}$ & $0.509^{* * *}$ & $\mathbf{0 . 0 1 8}^{\mathrm{ns}}$ & $90.963^{* * *}$ & $50180.942^{* * *}$ \\
\hline $\mathbf{S D} \times \mathbf{N} \times \mathbf{V}$ & $2.761^{* *}$ & $40.240^{* *}$ & $1.071^{* * *}$ & $7313.625^{* *}$ & $1.205^{* *}$ & $0.041^{* *}$ & $49.825^{* *}$ & $31362.277^{* * *}$ \\
\hline Error (C) & 0.172 & 6.054 & 0.143 & 683.347 & 0.054 & 0.011 & 6.970 & 4889.324 \\
\hline
\end{tabular}

$*$ and ${ }^{* *}$ Significant at 0.05 and 0.01 probability respectively and ns $=$ not significant . 


\subsection{Effect of sowing dates}

The results in Table(3) indicated that days to $50 \%$ flowering was increased from $(81.53,81.42)$ for early sowing at 15 October to $(83.27,83.16)$ for late sowing on $1^{\text {st }}$ December in the two successive seasons. Also, the results in Table (3) revealed that sowing on Nov $1^{\text {st }}$ showed the greatest value for seed yield / fedden (1050.13\& $1175.40 \mathrm{Kg}$ ) in the two successive seasons. This was attributed to the highest seed yield per plant $(43.76 \& 48.94 \mathrm{~g})$, as well as the highest values for yield components, i.e., No. of primary branches per plant, No. of Siliqua /plant, seeds/siliquas and 1000 seed weight. The results also cleared that early sowing on Oct.16 produced the least seed yield / fedden and per plant as well as the least values for plant seed yield and its components, followed by the latest sowing. However, sowing on Nov.16 ranked $2^{\text {nd }}$ followed by sowing on Dec. $1^{\text {st }}$.

Generally, these data proved that ,the planting at $1^{s t}$ November was the best for seed yield and its contributes. However the early and late sowing dates recorded a significant reduction in aforementioned characters. This may be attributed to weather conditions during the reproductive stage of the late sowing date which was very warm resulting in a reduction in number of primary branches and number of siliquas per plant. This trend decreased seed yield per plant. Also, in the late sowing dates, the plants were attacked by the cabbage aphid which caused a lot of damage in yield per plant. The result is in harmony with the results found by Jenkins and
Leitch (1986) Thomas et al. ( 1990 ) Taylor and Smith (1992) Leto et al. (1995), Starner et al (1996), Saini and Sidhu (1997), Ozer (2002) Sharief and Keshta (2002), Leilah, et al. ( 2003), Mohsen et al. (2004), Fathi et al. (2005) Gul and Ahmad (2007) Daneshian et al. (2008) and Shah and Rahman (2009).

\subsection{Effect of nitrogen fertilizer}

Data in Table (4) indicated that increasing N level from 30 to 60 or $90 \mathrm{Kg} /$ Fadden caused significant increase in all the studied traits in both seasons. Seed yield per Fadden increased by $39.30 \%$ and $51.50 \%$ on the same order as an average of both seasons. This was attributed with similar increase in seed yield per plant $40.00 \%$ and $53.30 \%$ as an average in both seasons. The increase in seed yield per plant as $\mathrm{N}$ level was increased was associated with an increase in plant yield components ,i.e. No.of. branches per plant, No.of. siliquas per plant, seeds/siliqua and 1000 seed weight. Also, increasing $\mathrm{N}$ level delayed flowering and increased plant height in both seasons.These findings reflected the important main role of nitrogen fertilizer for plant growth, yield and its components. And the important role of nitrogen in stimulating the meristematic activity and cell elongation of the plant and increasing photosynthesis activity of canola. These results are in agreement with those obtained by Singh and Srivastava (1986) Chauhan et al. (1993), Starner et al. (1996), Said and Keshta (1999), Sharief and Keshta (2000), Ozer (2002) Leilah et al. (2003) and Asfour (2006) .

Table ( 3 ) : Effect of sowing date on all the studied traits (over all nitrogen fertilizer levels and varieties) in 2007/2008 and $2008 / 2009$ seasons.

\begin{tabular}{|c|c|c|c|c|c|c|c|c|}
\hline Sowing date & $\begin{array}{c}\text { Days to } \\
50 \% \\
\text { flowering }\end{array}$ & $\begin{array}{c}\text { Plant } \\
\text { height } \\
(\mathrm{cm})\end{array}$ & $\begin{array}{c}\text { No . of } \\
\text { primary } \\
\text { branches / } \\
\text { Plant } \\
\end{array}$ & $\begin{array}{c}\text { No . of } \\
\text { siliquas / } \\
\text { plant }\end{array}$ & $\begin{array}{l}\text { No . of } \\
\text { seeds/ } \\
\text { siliqua }\end{array}$ & $\begin{array}{c}1000 \\
\text { seed } \\
\text { weight }\end{array}$ & $\begin{array}{c}\text { Seed } \\
\text { yield / } \\
\text { plant } \\
(\mathrm{g})\end{array}$ & $\begin{array}{c}\text { Seed } \\
\text { yield/ } \\
\text { fed }(\mathrm{kg})\end{array}$ \\
\hline \multicolumn{9}{|c|}{$2007 / 2008$ season } \\
\hline 15 October & 81.53 & 119.34 & 10.76 & 478.56 & 23.16 & 2.89 & 33.52 & 804.43 \\
\hline 1 November & 81.93 & 123.40 & 13.41 & 586.18 & 24.03 & 2.99 & 43.76 & 1050.13 \\
\hline 16 November & 82.91 & 121.96 & 12.54 & 579.52 & 23.68 & 2.86 & 40.68 & 976.21 \\
\hline 1 December & 83.27 & 122.50 & 12.34 & 553.20 & 23.91 & 2.80 & 38.20 & 918.35 \\
\hline LSD at 0.05 & 0.47 & 0.81 & 0.28 & 14.46 & 0.09 & 0.03 & 1.35 & 31.42 \\
\hline \multicolumn{9}{|c|}{ 2008/2009 season } \\
\hline 15 October & 81.42 & 117.06 & 11.70 & 436.42 & 23.19 & 2.86 & 30.72 & 737.28 \\
\hline 1 November & 82.07 & 123.55 & 13.31 & 619.04 & 24.17 & 3.12 & 48.94 & 1175.40 \\
\hline 16 November & 83.24 & 120.63 & 12.84 & 579.51 & 23.97 & 3.02 & 44.47 & 1059.44 \\
\hline 1 December & 83.16 & 117.20 & 11.83 & 526.08 & 23.14 & 2.87 & 36.72 & 881.45 \\
\hline LSD at 0.05 & 0.19 & 0.61 & 0.33 & 17.28 & 0.12 & 0.05 & 1.77 & 51.92 \\
\hline
\end{tabular}


Table (4) : Effect of nitrogen fertilizer levels on all the studied traits (over all sowing date and varieties) in $2007 / 2008$ and $2008 / 2009$ seasons.

\begin{tabular}{|c|c|c|c|c|c|c|c|c|}
\hline $\begin{array}{l}\text { Nitrogen } \\
\text { levels } \\
\text { Kg/Fed } \\
\end{array}$ & $\begin{array}{c}\text { Days to } \\
50 \% \\
\text { flowering } \\
\end{array}$ & $\begin{array}{c}\text { Plant } \\
\text { height } \\
(\mathrm{cm})\end{array}$ & $\begin{array}{c}\text { No. of } \\
\text { primary } \\
\text { branches/Plan }\end{array}$ & $\begin{array}{l}\text { No . of } \\
\text { siliquas } \\
\text { / plant }\end{array}$ & $\begin{array}{c}\text { No . of } \\
\text { seeds } \\
\text { /siliqua }\end{array}$ & $\begin{array}{c}1000 \\
\text { seed } \\
\text { weight }(g)\end{array}$ & $\begin{array}{c}\text { Seed } \\
\text { yield / } \\
\text { plant (g) }\end{array}$ & $\begin{array}{c}\text { Seed } \\
\text { yield/ } \\
\text { Fed (kg ) }\end{array}$ \\
\hline \multicolumn{9}{|c|}{$2007 / 2008$ season } \\
\hline 30 & 81.50 & 116.91 & 10.83 & 447.55 & 23.33 & 2.81 & 30.50 & 732.00 \\
\hline 60 & 82.67 & 123.04 & 12.58 & 575.50 & 23.81 & 2.93 & 41.41 & 995.04 \\
\hline 90 & 83.52 & 125.45 & 13.37 & 625.03 & 23.94 & 2.91 & 45.20 & 1084.80 \\
\hline LSD at0.05 & $\mathbf{0 . 3 3}$ & 0.65 & 0.28 & 10.08 & 0.06 & 0.04 & 0.79 & 13.81 \\
\hline \multicolumn{9}{|c|}{$2008 / 2009$ season } \\
\hline 30 & 81.03 & 116.12 & 11.37 & 459.47 & 23.10 & 2.71 & 30.52 & 726.39 \\
\hline 60 & 82.63 & 121.83 & 12.59 & 569.55 & 23.80 & 3.03 & 43.20 & 1037.67 \\
\hline 90 & 83.75 & 120.89 & 13.30 & 592.26 & 23.94 & 3.17 & 46.92 & 1126.12 \\
\hline LSD at0.05 & 0.13 & 0.99 & 0.14 & 8.94 & 0.09 & 0.04 & 0.96 & 24.67 \\
\hline
\end{tabular}

\subsection{Interaction between sowing dates and nitrogen fertilizer levels}

The interaction mean values presented in Table (5) showed that the best treatment was sowing at the first of November with $90 \mathrm{Kg} \mathrm{N} /$ fedden. This showed high mean values in most of the studied traits in the two seasons. Regarding seed yield per plant and seed yield /fed, the highest mean values were $(50.52 \mathrm{gm}$ and $1212.48 \mathrm{~K} \mathrm{~g})$ in the first season at the third sowing date with $90 \mathrm{KgN}$ added, but in the second season the best treatment was the second sowing date with $90 \mathrm{KgN}$ where it gave the highest seed yield and yield/fed $(57.48 \mathrm{~g}$ and $1379.53 \mathrm{Kg} / \mathrm{fed}$ ), respectively. However the lowest seed yield per plant were (23.63 and 22.23) at sowing date 15 October with $30 \mathrm{Kg} \mathrm{N} /$ Fadden in

Table (5 ) :The interaction mean values between sowing dates and nitrogen fertilizer levels ( over all varieties ) for all the studied traits in 2007/2008and2008/2008 seasons.

\begin{tabular}{|c|c|c|c|c|c|c|c|c|c|}
\hline Sowing date & $\begin{array}{c}\begin{array}{c}N \\
\text { levels } \\
\text { Kg/Fed }\end{array}\end{array}$ & $\begin{array}{c}\text { Days to } \\
50 \% \\
\text { flowering }\end{array}$ & $\begin{array}{l}\text { Plant } \\
\text { height } \\
(\mathrm{cm})\end{array}$ & $\begin{array}{c}\text { No . of } \\
\text { primary } \\
\text { branches / } \\
\text { Plant }\end{array}$ & $\begin{array}{l}\text { No . of } \\
\text { siliquas } \\
\text { / plant }\end{array}$ & $\begin{array}{l}\text { No . of } \\
\text { seeds/ } \\
\text { siliqua }\end{array}$ & $\begin{array}{c}1000 \text { seed } \\
\text { weight } \\
(\mathrm{g})\end{array}$ & $\begin{array}{c}\text { Seed } \\
\text { yield/ } \\
\text { plant } \\
\text { ( g) }\end{array}$ & $\begin{array}{c}\text { Seed } \\
\text { yield/ Fed } \\
(\mathrm{kg})\end{array}$ \\
\hline \multicolumn{10}{|c|}{$2007 / 2008$ season } \\
\hline \multirow{3}{*}{15 October } & 30 & 79.33 & 115.32 & 9.40 & 358.67 & 22.83 & 2.79 & 23.63 & 567.20 \\
\hline & 60 & 82.20 & 119.80 & 11.47 & 479.00 & 23.29 & 2.94 & 33.82 & 811.68 \\
\hline & 90 & 83.07 & 122.90 & 11.40 & 598.00 & 23.36 & 2.94 & 43.10 & 1034.40 \\
\hline \multirow{3}{*}{1 November } & 30 & 79.80 & 117.80 & 12.05 & 494.87 & 23.21 & 2.98 & 35.77 & 858.40 \\
\hline & 60 & 82.40 & 125.14 & 13.47 & 610.33 & 24.45 & 3.07 & 47.09 & 1130.10 \\
\hline & 90 & 83.60 & 127.25 & 14.70 & 653.33 & 24.43 & 2.91 & 48.41 & 1161.92 \\
\hline \multirow{3}{*}{16 November } & 30 & 82.27 & 119.15 & 11.00 & 489.80 & 23.25 & 2.73 & 32.33 & 775.84 \\
\hline & 60 & 82.40 & 119.73 & 12.73 & 576.25 & 23.36 & 2.83 & 39.18 & 940.32 \\
\hline & 90 & 84.07 & 126.99 & 13.87 & 672.51 & 24.41 & 3.01 & 50.52 & 1212.48 \\
\hline \multirow{3}{*}{1 December } & 30 & 82.80 & 115.37 & 10.85 & 446.89 & 24.03 & 2.72 & 30.27 & 726.56 \\
\hline & 60 & 83.67 & 127.50 & 12.64 & 636.42 & 24.15 & 2.89 & 45.51 & 1098.10 \\
\hline & 90 & 83.33 & 124.65 & 13.52 & 576.28 & 23.56 & 2.78 & 38.77 & 930.40 \\
\hline \multicolumn{2}{|l|}{ LSD at 0.05} & 0.65 & 1.31 & 0.57 & 20.16 & 0.12 & 0.06 & 1.58 & 39.07 \\
\hline \multicolumn{10}{|c|}{$2008 / 2009$ season } \\
\hline \multirow{3}{*}{15 October } & 30 & 79.67 & 113.82 & 10.20 & 353.80 & 23.05 & 2.58 & 22.23 & 533.44 \\
\hline & 60 & 81.60 & 117.47 & 11.37 & 470.40 & 23.03 & 2.91 & 33.03 & 792.64 \\
\hline & 90 & 83.00 & 119.91 & 13.53 & 485.05 & 23.48 & 3.10 & 36.91 & 885.76 \\
\hline \multirow{3}{*}{1 November } & 30 & 80.07 & 118.58 & 12.01 & 517.75 & 23.23 & 2.87 & 35.51 & 851.52 \\
\hline & 60 & 82.80 & 124.62 & 13.73 & 669.01 & 24.44 & 3.15 & 53.82 & 1295.16 \\
\hline & 90 & 83.33 & 127.44 & 14.20 & 670.36 & 24.85 & 3.35 & 57.48 & 1379.53 \\
\hline \multirow{3}{*}{16 November } & 30 & 82.20 & 117.24 & 11.89 & 494.77 & 23.41 & 2.81 & 34.71 & 809.36 \\
\hline & 60 & 82.73 & 123.18 & 12.93 & 598.03 & 24.33 & 3.05 & 46.52 & 1116.48 \\
\hline & 90 & 84.80 & 121.46 & 13.69 & 645.74 & 24.16 & 3.21 & 52.19 & 1252.48 \\
\hline \multirow{3}{*}{1 December } & 30 & 82.20 & 114.83 & 11.36 & 471.57 & 22.72 & 2.59 & 29.63 & 711.22 \\
\hline & 60 & 83.40 & 122.03 & 12.34 & 538.77 & 23.41 & 3.01 & 39.42 & 964.40 \\
\hline & 90 & 83.87 & 114.73 & 11.78 & 567.91 & 23.28 & 3.02 & 41.11 & 986.72 \\
\hline \multicolumn{2}{|l|}{ LSD at 0} & 0.27 & 1.99 & 0.27 & \begin{tabular}{ll|}
17.88 \\
\end{tabular} & 0.17 & 0.08 & 1.92 & $\begin{array}{l}49.34 \\
\end{array}$ \\
\hline
\end{tabular}


both seasons, respectively. Concerning earliness the best treatment was planting at the middle of October with $30 \mathrm{KgN} /$ fad where it gave values (79.33 and 79.67) days from sowing dates to $50 \%$ flowering in both seasons, respectively. On the other hand, increasing nitrogen level up to $90 \mathrm{Kg}$ $\mathrm{N} /$ fed with planting at the middle of November caused the most delay in flowering to (84.07 and 84.80 days) in both season respectively. These results are in harmony with the results found by Singh and Singh ( 1984 ) Ozer (2002) Leilah, et al. (2003).

\subsection{Effect of varieties}

Data in Table (6) showed significant differences among the used varieties in all the studied characters in both seasons.Serw-4 was the most early in flowering, however it showed the tallest plants. Also, Serw-4 produced the highest seed yield per feddan (1551.33 and $1717.27 \mathrm{Kg}$ )in the two successive seasons. This was due to highest seed yield per plant (64.63and71.56g)in both seasons, respectively . The highest seed yield per plant of Serw-4 was attributed to the highest plant yield components, i.e. No. of. Branches per plant ,No.of. siliquas /plant, number of seeds/ siliqua and 1000 seed weight. Duplo variety ranked the second with significant difference from Serw-4 and Pactol variety which ranked third. The differences among the varieties could be due to the differences in the genetic constitution and their response to environmental conditions. These results are in harmony with the results by obtained Asfour, (2006).

3.5. Interaction between sowing dates and varieties

Data in Table $(7 \mathrm{a} \& b)$ cleared that the highest seed yield per fadden was obtained by sowing
Serw-4 on Nov. $1^{\text {st }}$ in both seasons with significant difference from other all treatments. This may be due to that this variety was the most adapted for the conditions of experimental site because this treatment produced tallest plants, most early plants in flowering, plants produced more branches and siliquas per plant, highest seed number per siliqua in both seasons.

\subsection{Effect of interaction between nitrogen} levels and varieties

The interaction mean values are summarized in Table(8). Data obtained showed that this interaction had significant effect on all the studied traits in both seasons. This means that the response of used varieties to increasing $\mathrm{N}$ level was different. Therefore, Serw-4 variety was more efficient in fertilizer use than others because it produced the highest values for all traits at all $\mathrm{N}$ levels. Also, it produced highest seed yield per plant and per faddan as well as highest values for other traits when $90 \mathrm{KgN} /$ faddan was added in both seasons. On other hand Sedo variety showed the least valus for all traits with addition of $30 \mathrm{KgN} /$ faddan in both seasons. These results are in harmony with the results found by Asfour (2006).

3.7.The interaction among sowing dates, nitrogen fertilizer levels, and varieties

Statistical analysis data in Table (2) showed high significant differences among all the tested treatments for all studied traits in both studied seasons. Table $(9 \mathrm{a} \& \mathrm{~b})$ cleared that this interaction had significant effect on all studied traits in both seasons. Data indicated that the highest seed yield per fadden was obtained when Serw-4 variety was sown on Nov. $1^{\text {st }}$ and fertilized with $90 \mathrm{Kg}$ $\mathrm{N} /$ fadden. This was true in both seasons. This

Table (6) : Mean values of varieties on all traits (over all nitrogen fertilizer levels and sowing date) in 2007/2008and 2008/2009seasons.

\begin{tabular}{|c|c|c|c|c|c|c|c|c|}
\hline Varieties & \begin{tabular}{|c|} 
Days to \\
$50 \%$ \\
flowering
\end{tabular} & $\begin{array}{l}\text { Plant } \\
\text { height } \\
(\mathrm{cm})\end{array}$ & $\begin{array}{c}\text { No . of primary } \\
\text { branches / } \\
\text { Plant }\end{array}$ & $\begin{array}{c}\text { No . of } \\
\text { siliquas / } \\
\text { plant }\end{array}$ & $\begin{array}{l}\text { No . of } \\
\text { seeds/ } \\
\text { siliqua }\end{array}$ & $\begin{array}{l}1000 \text { seed } \\
\text { weight }(\mathrm{g})\end{array}$ & $\begin{array}{l}\text { Seed yield/ } \\
\text { plant } \\
(\mathrm{g})\end{array}$ & $\begin{array}{c}\text { Seed } \\
\text { yield/ } \\
\text { Fed }(\mathbf{k g})\end{array}$ \\
\hline \multicolumn{9}{|c|}{ 2007/2008 season } \\
\hline Sedo & 83.58 & 117.53 & 10.19 & 452.96 & 22.81 & 2.64 & 27.56 & 661.47 \\
\hline Duplo & 85.19 & 122.58 & 12.41 & 564.35 & 24.20 & 3.14 & 43.11 & 1036.53 \\
\hline Serw -4 & 75.39 & 132.42 & 15.44 & 722.20 & 25.67 & 3.46 & 64.63 & 1551.33 \\
\hline Pactol & 84.19 & 118.29 & 11.47 & 509.62 & 22.92 & 2.61 & 30.66 & 735.93 \\
\hline Drakkar & 83.69 & 118.17 & 11.79 & 497.68 & 22.87 & 2.55 & 29.21 & 701.13 \\
\hline LSD at0.05 & 0.35 & 0.80 & 0.28 & 13.13 & 0.08 & 0.04 & 1.06 & 25.81 \\
\hline \multicolumn{9}{|c|}{$2008 / 2009$ season } \\
\hline Sedo & 83.81 & 116.30 & 11.28 & 420.91 & 22.50 & 2.66 & 25.85 & 610.37 \\
\hline Duplo & 84.94 & 121.80 & 13.01 & 559.23 & 24.17 & 3.20 & 43.86 & 1052.60 \\
\hline Serw -4 & 74.78 & 129.16 & 15.20 & 774.43 & 25.63 & 3.55 & 71.56 & 1717.27 \\
\hline Pactol & 84.06 & 116.82 & 11.53 & 472.64 & 23.18 & 2.74 & 30.45 & 731.01 \\
\hline Drakkar & 84.78 & 113.37 & 11.08 & 474.11 & 22.61 & 2.70 & 29.35 & 705.72 \\
\hline LSD at 0.05 & 0.19 & 1.14 & 0.18 & 12.20 & 0.10 & 0.05 & 1.23 & 32.63 \\
\hline
\end{tabular}


Table ( 7 a): The interaction mean values between sowing date and varieties (over all nitrogen fertilizer levels) for all the studied traits in $2007 / 2008$ season.

\begin{tabular}{|c|c|c|c|c|c|c|c|c|c|}
\hline $\begin{array}{c}\text { Sowing } \\
\text { date }\end{array}$ & Varieties & $\begin{array}{c}\text { Days to } \\
50 \% \\
\text { flowering }\end{array}$ & $\begin{array}{c}\text { Plant } \\
\text { height } \\
\text { (cm) }\end{array}$ & $\begin{array}{c}\text { No . of } \\
\text { primary } \\
\text { branches / } \\
\text { Plant }\end{array}$ & $\begin{array}{c}\text { No . of } \\
\text { siliquas / } \\
\text { plant }\end{array}$ & $\begin{array}{c}\text { No . of } \\
\text { seeds } \\
\text { /siliqua }\end{array}$ & $\begin{array}{c}1000 \\
\text { seed } \\
\text { weight } \\
(\mathrm{g}) \\
\end{array}$ & $\begin{array}{c}\text { Seed } \\
\text { yield / } \\
\text { plant (g) }\end{array}$ & $\begin{array}{c}\text { Seed } \\
\text { yield/ } \\
\text { Fed } \\
(\mathbf{k g})\end{array}$ \\
\hline \multicolumn{10}{|c|}{$2007 / 2008$ season } \\
\hline \multirow{5}{*}{15 October } & Sedo & 82.33 & 116.84 & 8.39 & 380.00 & 22.27 & 2.71 & 23.12 & 554.93 \\
\hline & Duplo & 84.33 & 121.33 & 10.22 & 491.11 & 24.02 & 3.19 & 38.05 & 913.33 \\
\hline & Serw -4 & 75.22 & 128.46 & 14.67 & 640.00 & 25.36 & 3.44 & 56.62 & 1358.93 \\
\hline & Pactol & 83.67 & 114.11 & 9.72 & 443.89 & 22.07 & 2.54 & 24.96 & 598.93 \\
\hline & Drakkar & 82.11 & 115.96 & 10.78 & 437.78 & 22.09 & 2.57 & 24.83 & 596.00 \\
\hline \multirow{5}{*}{1 November } & Sedo & 83.00 & 120.42 & 11.42 & 498.00 & 22.78 & 2.68 & 30.40 & 729.60 \\
\hline & Duplo & 84.67 & 124.10 & 13.53 & 614.56 & 24.67 & 3.27 & 49.20 & 1180.80 \\
\hline & Serw - 4 & 74.33 & 134.14 & 16.58 & 770.00 & 26.04 & 3.68 & 73.93 & 1774.40 \\
\hline & Pactol & 83.67 & 119.67 & 12.36 & 535.00 & 23.33 & 2.73 & 34.13 & 819.20 \\
\hline & Drakkar & 84.00 & 118.66 & 13.16 & 513.33 & 23.33 & 2.58 & 31.11 & 746.67 \\
\hline \multirow{5}{*}{16 November } & Sedo & 84.33 & 117.81 & 10.33 & 496.80 & 22.78 & 2.67 & 30.64 & 735.47 \\
\hline & Duplo & 86.22 & 121.78 & 13.00 & 589.28 & 24.07 & 3.04 & 43.31 & 1039.47 \\
\hline & Serw -4 & 75.67 & 133.33 & 15.97 & 744.10 & 26.00 & 3.39 & 66.01 & 1584.27 \\
\hline & Pactol & 84.67 & 119.00 & 11.84 & 542.59 & 22.84 & 2.62 & 32.68 & 784.27 \\
\hline & Drakkar & 83.67 & 117.87 & 11.53 & 524.84 & 22.69 & 2.56 & 30.73 & 737.60 \\
\hline \multirow{5}{*}{1 December } & Sedo & 84.67 & 115.06 & 10.60 & 437.06 & 23.42 & 2.50 & 26.08 & 625.87 \\
\hline & Duplo & 85.56 & 123.12 & 12.89 & 562.46 & 24.04 & 3.08 & 41.86 & 1012.53 \\
\hline & Serw -4 & 76.33 & 133.73 & 14.56 & 734.71 & 25.29 & 3.34 & 61.98 & 1487.73 \\
\hline & Pactol & 84.78 & 120.40 & 11.96 & 517.01 & 23.42 & 2.56 & 30.89 & 741.33 \\
\hline & Drakkar & 85.00 & 120.21 & 11.69 & 514.74 & 23.38 & 2.51 & 30.18 & 724.27 \\
\hline \multicolumn{2}{|c|}{ LSD at 0.05} & 0.71 & 1.60 & 0.56 & 26.27 & 0.17 & 0.08 & 2.12 & 51.62 \\
\hline
\end{tabular}

Table ( 7 b ) :The interaction mean values between sowing date and varieties ( over all nitrogen fertilizer levels) for all the studied traits in 2008/2009seasons.

\begin{tabular}{|c|c|c|c|c|c|c|c|c|c|}
\hline $\begin{array}{c}\text { Sowing } \\
\text { date }\end{array}$ & Varieties & $\begin{array}{c}\text { Days to } \\
50 \% \\
\text { flowering }\end{array}$ & $\begin{array}{c}\text { Plant } \\
\text { height } \\
(\mathrm{cm})\end{array}$ & $\begin{array}{c}\text { No . of } \\
\text { primary } \\
\text { branches / } \\
\text { Plant }\end{array}$ & $\begin{array}{c}\text { No . of } \\
\text { siliquas / } \\
\text { plant }\end{array}$ & $\begin{array}{l}\text { No. of } \\
\text { seeds/ } \\
\text { siliqua }\end{array}$ & $\begin{array}{c}1000 \\
\text { seed } \\
\text { weight }(\mathrm{g})\end{array}$ & $\begin{array}{c}\text { Seed } \\
\text { yield } / \\
\text { plant }(g)\end{array}$ & $\begin{array}{c}\text { Seed } \\
\text { yield/ } \\
\text { Fed(kg) }\end{array}$ \\
\hline \multicolumn{10}{|c|}{ 2008/2009 season } \\
\hline \multirow{5}{*}{15 October } & Sedo & 82.33 & 116.12 & 10.69 & 307.01 & 22.18 & 2.50 & 17.33 & 415.73 \\
\hline & Duplo & 83.56 & 119.92 & 12.61 & 493.77 & 23.84 & 3.14 & 37.42 & 897.87 \\
\hline & Serw -4 & 74.67 & 125.19 & 14.39 & 602.62 & 24.84 & 3.44 & 52.65 & 1263.47 \\
\hline & Pactol & 83.22 & 114.63 & 10.37 & 372.78 & 11.98 & 2.62 & 22.71 & 545.07 \\
\hline & Drakkar & 83.33 & 109.46 & 10.46 & 405.90 & 22.09 & 2.61 & 23.51 & 564.27 \\
\hline \multirow{5}{*}{1 November } & Sedo & 83.33 & 117.20 & 12.02 & 511.48 & 22.53 & 2.84 & 32.92 & 790.15 \\
\hline & Duplo & 84.33 & 125.78 & 14.38 & 638.17 & 24.87 & 3.36 & 53.16 & 1275.73 \\
\hline & Serw -4 & 73.67 & 134.10 & 15.98 & 885.89 & 25.87 & 3.66 & 84.99 & 2038.67 \\
\hline & Pactol & 84.11 & 121.18 & 12.24 & 539.26 & 23.82 & 2.87 & 37.18 & 893.07 \\
\hline & Drakkar & 84.89 & 119.48 & 11.94 & 520.42 & 23.78 & 2.90 & 36.43 & 879.40 \\
\hline \multirow{5}{*}{16 November } & Sedo & 84.33 & 118.67 & 11.68 & 459.54 & 23.00 & 2.73 & 29.38 & 665.47 \\
\hline & Duplo & 85.56 & 121.33 & 13.10 & 591.44 & 24.40 & 3.30 & 47.95 & 1150.93 \\
\hline & Serw -4 & 76.22 & 130.56 & 15.92 & 867.73 & 26.47 & 3.60 & 82.94 & 1990.67 \\
\hline & Pactol & 84.56 & 118.62 & 12.17 & 492.50 & 23.62 & 2.80 & 32.88 & 789.33 \\
\hline & Drakkar & 85.56 & 113.96 & 11.33 & 486.34 & 22.36 & 2.67 & 29.20 & 700.80 \\
\hline \multirow{5}{*}{1 December } & Sedo & 85.22 & 113.20 & 10.74 & 405.60 & 22.29 & 2.57 & 23.75 & 570.14 \\
\hline & Duplo & 86.33 & 120.17 & 11.97 & 513.54 & 23.56 & 3.01 & 36.90 & 885.87 \\
\hline & Serw -4 & 74.56 & 126.80 & 14.50 & 741.48 & 25.36 & 3.49 & 65.67 & 1576.27 \\
\hline & Pactol & 84.33 & 112.84 & 11.33 & 486.04 & 22.29 & 2.68 & 29.02 & 696.57 \\
\hline & Drakkar & 85.33 & 112.99 & 10.60 & 483.76 & 22.20 & 2.62 & 28.27 & 678.40 \\
\hline \multicolumn{2}{|c|}{ LSD at 0.05} & 0.39 & 1.34 & 0.35 & 24.40 & 0.22 & 0.10 & 2.46 & 65.27 \\
\hline
\end{tabular}


Table ( 8 ):The interaction mean values between nitrogen fertilizer levels and varieties (over all the sowing date) for all the studied traits in 2007/2008and 2008/2009 seasons.

\begin{tabular}{|c|c|c|c|c|c|c|c|c|c|}
\hline $\begin{array}{l}\text { N levels } \\
\text { Kg/ Fed }\end{array}$ & Varieties & $\begin{array}{c}\text { Days to } \\
50 \% \\
\text { flowering }\end{array}$ & $\begin{array}{c}\text { Plant } \\
\text { height } \\
(\mathrm{cm})\end{array}$ & $\begin{array}{c}\text { No . of } \\
\text { primary } \\
\text { branches/ } \\
\text { Plant }\end{array}$ & $\begin{array}{c}\text { No . of } \\
\text { siliquas / } \\
\text { plant }\end{array}$ & $\begin{array}{l}\text { No . of } \\
\text { seeds / } \\
\text { siliqua }\end{array}$ & $\begin{array}{c}1000 \\
\text { seed } \\
\text { weight } \\
(\mathrm{g})\end{array}$ & $\begin{array}{c}\text { Seed } \\
\text { yield/ } \\
\text { plant } \\
(\mathrm{g})\end{array}$ & $\begin{array}{c}\text { Seed } \\
\text { yield/ } \\
\text { Fed } \\
(\mathbf{k g})\end{array}$ \\
\hline \multicolumn{10}{|c|}{$2007 / 2008$ season } \\
\hline \multirow{5}{*}{30} & Sedo & 81.50 & 111.49 & 8.91 & 351.97 & 22.05 & 2.52 & 19.59 & 470.20 \\
\hline & Duplo & 83.50 & 118.00 & 11.08 & 462.84 & 24.03 & 3.07 & 34.43 & 826.40 \\
\hline & Serw -4 & 74.42 & 124.63 & 13.43 & 596.25 & 25.52 & 3.33 & 50.85 & 1220.60 \\
\hline & Pactol & 83.75 & 115.38 & 10.17 & 419.21 & 22.53 & 2.59 & 24.52 & 588.60 \\
\hline & Drakkar & 82.08 & 115.05 & 10.54 & 407.50 & 22.52 & 2.51 & 23.09 & 554.20 \\
\hline \multirow{5}{*}{60} & Sedo & 84.00 & 119.83 & 10.69 & 483.44 & 22.70 & 2.70 & 29.57 & 709.80 \\
\hline & Duplo & 85.50 & 123.83 & 12.52 & 583.42 & 24.55 & 3.25 & 46.63 & 1125.00 \\
\hline & Serw -4 & 75.42 & 133.36 & 15.68 & 748.44 & 25.67 & 3.47 & 66.74 & 1601.80 \\
\hline & Pactol & 84.17 & 118.75 & 12.01 & 536.08 & 23.13 & 2.63 & 32.56 & 781.40 \\
\hline & Drakkar & 84.25 & 119.45 & 11.99 & 526.13 & 23.02 & 2.60 & 31.55 & 757.20 \\
\hline \multirow{5}{*}{90} & Sedo & 85.25 & 121.27 & 10.96 & 523.47 & 23.68 & 2.70 & 33.52 & 804.40 \\
\hline & Duplo & 86.58 & 125.93 & 13.63 & 646.79 & 24.02 & 3.10 & 48.26 & 1158.20 \\
\hline & Serw -4 & 76.33 & 139.26 & 17.23 & 821.92 & 25.83 & 3.58 & 76.32 & 1831.60 \\
\hline & Pactol & 84.67 & 120.76 & 12.23 & 573.57 & 23.08 & 2.62 & 34.81 & 837.80 \\
\hline & Drakkar & 84.75 & 120.02 & 12.83 & 559.40 & 23.08 & 2.54 & 32.99 & 792.00 \\
\hline \multicolumn{2}{|c|}{ LSD at 0.05} & 0.61 & 0.97 & 0.49 & 22.75 & 0.15 & 0.07 & 1.83 & 44.71 \\
\hline \multicolumn{10}{|c|}{ 2008/2009 season } \\
\hline \multirow{5}{*}{30} & Sedo & 82.08 & 112.98 & 10.23 & 319.13 & 22.17 & 2.40 & 17.35 & 386.71 \\
\hline & Duplo & 83.58 & 118.59 & 11.65 & 468.62 & 23.48 & 2.90 & 32.12 & 771.00 \\
\hline & Serw -4 & 73.42 & 123.32 & 14.08 & 693.73 & 24.88 & 3.31 & 57.63 & 1382.40 \\
\hline & Pactol & 82.92 & 113.75 & 10.57 & 405.45 & 22.78 & 2.47 & 22.77 & 546.63 \\
\hline & Drakkar & 83.17 & 111.94 & 10.30 & 410.43 & 22.20 & 2.49 & 22.72 & 545.20 \\
\hline \multirow{5}{*}{60} & Sedo & 84.00 & 118.37 & 11.47 & 462.85 & 22.80 & 2.72 & 29.00 & 696.01 \\
\hline & Duplo & 84.92 & 124.01 & 13.14 & 613.73 & 24.35 & 3.25 & 48.77 & 1170.80 \\
\hline & Serw -4 & 74.92 & 134.06 & 15.47 & 801.24 & 25.83 & 3.63 & 76.07 & 1825.60 \\
\hline & Pactol & 84.33 & 117.53 & 11.74 & 479.22 & 23.27 & 2.83 & 31.57 & 758.40 \\
\hline & Drakkar & 85.00 & 115.16 & 11.15 & 488.23 & 22.77 & 2.73 & 30.57 & 737.55 \\
\hline \multirow{5}{*}{90} & Sedo & 85.33 & 117.54 & 12.15 & 480.74 & 22.53 & 2.87 & 31.19 & 748.41 \\
\hline & Duplo & 86.33 & 122.80 & 14.25 & 595.34 & 24.67 & 3.46 & 50.67 & 1216.00 \\
\hline & Serw -4 & 76.00 & 130.10 & 16.03 & 828.32 & 26.18 & 3.71 & 80.99 & 1943.80 \\
\hline & Pactol & 84.92 & 119.17 & 12.27 & 533.27 & 23.48 & 2.93 & 37.00 & 888.00 \\
\hline & Drakkar & 86.17 & 114.82 & 11.80 & 523.66 & 22.85 & 2.87 & 34.77 & 834.40 \\
\hline \multicolumn{2}{|c|}{ LSD at 0.05} & 0.34 & 1.99 & 0.35 & 21.13 & 0.19 & 0.09 & 2.13 & 56.52 \\
\hline
\end{tabular}


Table ( 9 a ) : The interaction mean values among nitrogen fertilizer levels, sowing date and varieties on all the studied traits in both seasons.

\begin{tabular}{|c|c|c|c|c|c|c|c|c|c|c|c|c|c|c|c|c|c|c|c|c|c|}
\hline \multirow[t]{2}{*}{ Sowing date } & \multirow[t]{2}{*}{$\begin{array}{l}\text { N levels } \\
\text { Kg/Fed }\end{array}$} & \multicolumn{5}{|c|}{ Days to $50 \%$ flowering } & \multicolumn{5}{|c|}{ Plant height $(\mathrm{cm})$} & \multicolumn{5}{|c|}{ No . of primary branches / Plant } & \multicolumn{5}{|c|}{ No . of siliquas / plant } \\
\hline & & V1 & V2 & V3 & V4 & V5 & V1 & V2 & V3 & V4 & V5 & V1 & V2 & V3 & V4 & V5 & V1 & V2 & V3 & V4 & V5 \\
\hline \multicolumn{22}{|c|}{$2007 / 2008$ season } \\
\hline \multirow{3}{*}{15 October } & 30 & 80.00 & 82.67 & 74.67 & 83.00 & 76.33 & 114.20 & $\mathbf{1 1 7 . 3 3}$ & 125.03 & 108.50 & 111.53 & 7.83 & 9.67 & 12.67 & 7.83 & 9.00 & 226.67 & 346.67 & 520.00 & 363.33 & 336.67 \\
\hline & 60 & 82.00 & 84.33 & 75.12 & 84.00 & 85.00 & 117.50 & 121.67 & 127.00 & 115.50 & 117.33 & 9.33 & 11.33 & 14.67 & 10.67 & 11.33 & $\mathbf{3 5 3 . 3 3}$ & 450.00 & 643.33 & 475.00 & 473.33 \\
\hline & 90 & 85.00 & 86.00 & 75.33 & 84.00 & $\mathbf{8 5 . 0 0}$ & $\mathbf{1 1 8 . 8 3}$ & 125.00 & $\mathbf{1 3 3 . 3 3}$ & 118.33 & 119.00 & 8.00 & 9.67 & 16.67 & 10.67 & 12.00 & 560.00 & 676.67 & 756.67 & 493.33 & $\mathbf{5 0 3 . 3 3}$ \\
\hline \multirow{3}{*}{ 1November } & 30 & 80.00 & 82.00 & 72.00 & 82.00 & $\mathbf{8 3 . 0 0}$ & 112.00 & 118.67 & 123.33 & 117.33 & 117.67 & 10.27 & 11.33 & 14.00 & 12.00 & 12.67 & 434.00 & 543.67 & 626.67 & 430.00 & 440.00 \\
\hline & 60 & 84.00 & 85.00 & 75.00 & 84.00 & 84.00 & 123.83 & 125.30 & 137.43 & 120.33 & 118.80 & 12.00 & 13.00 & 17.33 & 12.67 & 12.33 & 533.33 & 613.33 & 776.67 & 575.00 & 553.33 \\
\hline & 90 & 85.00 & 87.00 & 76.00 & 85.00 & 85.00 & 125.43 & 128.33 & 141.67 & 121.33 & 119.50 & 12.00 & 16.27 & 18.40 & 12.40 & 14.47 & 526.67 & 686.67 & 906.67 & 600.00 & 546.67 \\
\hline \multirow{3}{*}{16 November } & 30 & 83.00 & 85.33 & 75.00 & 85.00 & 83.00 & 114.77 & 119.00 & 125.00 & 119.00 & 118.00 & 9.00 & 11.67 & 14.67 & 9.67 & 10.00 & 410.00 & 494.00 & 675.00 & 436.67 & 433.33 \\
\hline & 60 & 85.00 & 86.00 & 74.00 & 84.00 & 83.00 & 117.33 & 121.33 & 126.67 & 116.00 & 117.33 & 10.00 & 13.33 & 16.00 & 12.33 & 12.00 & 510.00 & 620.00 & 703.33 & 547.23 & 500.70 \\
\hline & 90 & 85.00 & 87.33 & 78.00 & 85.00 & 85.00 & 121.33 & 125.00 & 148.33 & 122.00 & 118.27 & 12.00 & 14.00 & 17.23 & 13.53 & 12.60 & 570.40 & 653.83 & 853.97 & 643.87 & 640.50 \\
\hline \multirow{3}{*}{1 December } & 30 & 83.00 & 84.00 & 76.00 & 85.00 & 86.00 & 105.00 & 117.00 & 125.17 & 116.67 & 113.00 & 8.53 & 11.67 & 12.37 & 11.20 & 10.50 & 337.23 & 467.03 & 563.33 & 446.83 & 420.00 \\
\hline & 60 & 85.00 & 86.67 & 77.00 & 84.67 & 85.00 & 120.67 & 127.00 & 142.33 & 123.17 & 124.33 & 11.43 & 12.40 & 14.70 & 12.37 & 12.30 & 537.10 & 650.33 & 870.43 & 547.10 & 577.13 \\
\hline & 90 & 86.00 & 86.00 & 76.00 & 84.67 & 84.00 & 119.50 & 125.37 & 133.70 & 121.37 & 123.30 & 11.83 & 14.60 & 16.60 & 12.30 & 12.27 & 436.83 & 570.00 & 770.37 & $\mathbf{5 5 7 . 1 0}$ & 547.10 \\
\hline \multicolumn{2}{|c|}{ LSD at 0.05} & \multicolumn{5}{|c|}{1.23} & \multicolumn{5}{|c|}{2.77} & \multicolumn{5}{|c|}{0.97} & \multicolumn{5}{|c|}{45.49} \\
\hline \multicolumn{22}{|c|}{ 2008/2009 season } \\
\hline \multirow{3}{*}{15 October } & 30 & 80.33 & 81.67 & 74.67 & 82.00 & 79.67 & 115.00 & 118.50 & 122.83 & 111.63 & 101.11 & 9.33 & 11.50 & 12.67 & 8.33 & 9.17 & 227.03 & 463.97 & 513.73 & 213.87 & $\mathbf{3 5 0 . 4 0}$ \\
\hline & 60 & 82.00 & 83.67 & 74.00 & 83.67 & 84.67 & 116.63 & 119.27 & 126.07 & 113.60 & 111.77 & 10.33 & 12.17 & 14.17 & 10.43 & 9.77 & 337.03 & 477.03 & 650.33 & 460.63 & 426.97 \\
\hline & 90 & 84.67 & 85.33 & 75.33 & 84.00 & 85.67 & 116.73 & 122.00 & 126.67 & 118.67 & 115.50 & 12.40 & 14.17 & 16.33 & 12.33 & 12.43 & 356.97 & 540.30 & 643.80 & 443.83 & 440.33 \\
\hline \multirow{3}{*}{ 1November } & 30 & 80.00 & 82.00 & 71.00 & 83.00 & 84.33 & 112.83 & 122.50 & 127.13 & 115.50 & 114.93 & 11.27 & 12.37 & 14.47 & 11.40 & 10.53 & 460.43 & 480.43 & 760.33 & 470.43 & 417.13 \\
\hline & 60 & 85.00 & 85.00 & 75.00 & 84.67 & 84.33 & 116.60 & 125.27 & 136.83 & 122.53 & 121.87 & 12.27 & 14.40 & 16.67 & 12.67 & 12.67 & 526.97 & 767.03 & 923.70 & 550.30 & 577.07 \\
\hline & 90 & 85.00 & 86.00 & 75.00 & 84.67 & 86.00 & 122.17 & 129.57 & 138.33 & 125.50 & 121.63 & 12.53 & 16.37 & 16.80 & 12.67 & 12.63 & 547.03 & 667.03 & 973.63 & 597.03 & 567.07 \\
\hline \multirow{3}{*}{16 November } & 30 & 83.00 & 84.67 & 75.00 & 83.67 & 84.67 & 115.60 & 119.03 & 121.67 & 115.20 & 114.70 & 10.83 & 12.00 & 14.57 & 11.50 & 10.57 & 327.07 & 473.67 & 784.03 & 462.00 & 427.07 \\
\hline & 60 & 84.00 & 85.00 & 75.67 & 84.00 & 85.00 & 120.23 & 121.33 & 136.67 & 120.67 & 117.00 & 11.57 & 12.60 & 16.50 & 12.33 & 11.67 & 510.43 & 663.73 & 883.83 & 463.77 & 468.40 \\
\hline & 90 & 86.00 & 87.00 & 78.00 & 86.00 & 87.00 & 120.17 & 123.63 & 133.33 & 120.00 & 110.17 & 12.63 & 14.70 & 16.70 & 12.67 & 11.77 & 541.13 & 636.93 & 935.33 & 551.73 & 563.57 \\
\hline \multirow{3}{*}{1 December } & 30 & 85.00 & 86.00 & 73.00 & 83.00 & 84.00 & 108.50 & 114.33 & 121.67 & 112.67 & 117.00 & 9.49 & 10.73 & 14.63 & 11.03 & 10.93 & 262.00 & 456.40 & 716.83 & 475.50 & 447.10 \\
\hline & 60 & 85.00 & 86.00 & 75.00 & 85.00 & 86.00 & 120.00 & 130.17 & 136.67 & 113.33 & 110.00 & 11.70 & 13.40 & 14.57 & 11.53 & 10.50 & 476.97 & 547.13 & 747.10 & 442.17 & 480.50 \\
\hline & 90 & 85.67 & 87.00 & 75.67 & 85.00 & 86.00 & 111.10 & 116.00 & 122.07 & 112.53 & 111.97 & 11.03 & 11.77 & 14.30 & 11.43 & 10.37 & 477.83 & 537.10 & 760.50 & 540.47 & 523.67 \\
\hline \multicolumn{2}{|c|}{ LSD at 0.05} & \multicolumn{5}{|c|}{0.29} & & & 0.15 & & & & & 3.68 & & & & & 89.41 & & \\
\hline
\end{tabular}

V1,2,3,4,5 = Sedo, Duplo , Serw-4, Pactol, and Drakkar varieties respectively . 
Table ( 9 b ): The interaction mean values among nitrogen fertilizer levels, sowing date and varieties on all the studied traits in both seasons.

\begin{tabular}{|c|c|c|c|c|c|c|c|c|c|c|c|c|c|c|c|c|c|c|c|c|c|}
\hline \multirow[t]{2}{*}{ Sowing date } & \multirow{2}{*}{$\begin{array}{l}\mathrm{N} \text { levels } \\
\mathrm{Kg} \text { /Fed }\end{array}$} & \multicolumn{5}{|c|}{ No . of seeds /siliqua } & \multicolumn{5}{|c|}{1000 seed weight $(\mathrm{g})$} & \multicolumn{5}{|c|}{ Seed yield / plant ( g) } & \multicolumn{5}{|c|}{ Seed yield/ Fed $(\mathrm{kg})$} \\
\hline & & V1 & V2 & V3 & V4 & V5 & V1 & $\mathrm{V2}$ & V3 & V4 & V5 & V1 & V2 & V3 & V4 & V5 & V1 & V2 & V3 & V4 & V5 \\
\hline \multicolumn{22}{|c|}{ 2007/2008 season } \\
\hline \multirow[b]{3}{*}{15 October } & 30 & 22.00 & 24.07 & 24.00 & 22.00 & 22.07 & 2.64 & 2.97 & 3.27 & 2.53 & 2.57 & 13.30 & 24.77 & 40.73 & 20.27 & 19.10 & 319.20 & 594.40 & 977.60 & 486.40 & 458.40 \\
\hline & 60 & 22.40 & 24.00 & 26.07 & 22.00 & 22.00 & 2.80 & 3.27 & 3.40 & 2.57 & 2.67 & 22.17 & 35.27 & 57.03 & 26.87 & 27.77 & 532.00 & 846.40 & 1368.80 & 644.80 & 666.40 \\
\hline & 90 & 22.40 & 24.00 & 26.00 & 22.20 & 22.20 & 2.70 & 3.33 & 3.67 & 2.53 & 2.47 & 33.90 & 54.13 & 72.10 & 27.73 & 27.63 & 813.60 & 1299.20 & 1730.40 & 665.60 & 663.20 \\
\hline \multirow[b]{3}{*}{ 1November } & 30 & 22.00 & 24.00 & 26.07 & 22.00 & 22.00 & 2.70 & $\mathbf{3 . 5 0}$ & 3.57 & 2.67 & 2.47 & 25.80 & 45.63 & 58.27 & 25.23 & 23.90 & 619.20 & 1095.20 & 1398.40 & 605.60 & 573.60 \\
\hline & 60 & 22.20 & 26.00 & 26.07 & 24.00 & 24.00 & 2.77 & $\mathbf{3 . 5 0}$ & 3.73 & 2.73 & 2.60 & 32.77 & 55.80 & 75.57 & 36.77 & 34.53 & 786.40 & 1339.20 & 1813.60 & 882.40 & 828.80 \\
\hline & 90 & 24.13 & 24.00 & 26.00 & 24.00 & 24.00 & 2.57 & 2.80 & 3.73 & 2.80 & 2.67 & 32.63 & 46.17 & 87.97 & 40.40 & 34.90 & 783.20 & 1108.00 & 2111.20 & 969.60 & 837.60 \\
\hline \multirow[b]{3}{*}{ 16November } & 30 & 22.13 & 24.07 & 26.00 & 22.07 & 22.00 & 2.47 & 2.90 & 3.17 & 2.63 & 2.50 & 22.40 & 34.43 & 55.57 & 25.39 & 23.83 & $\mathbf{5 3 7 . 6 0}$ & 826.40 & 1333.60 & 609.60 & 572.00 \\
\hline & 60 & 22.20 & 24.13 & 26.00 & 22.40 & 22.07 & 2.63 & 3.00 & $\mathbf{3 . 3 3}$ & 2.60 & 2.57 & 29.83 & 44.80 & 61.03 & 31.88 & 28.37 & 716.00 & 1075.20 & 1464.80 & 764.80 & 680.80 \\
\hline & 90 & 24.00 & 24.00 & 26.00 & 24.07 & 24.00 & 2.90 & 3.23 & 3.67 & 2.63 & 2.60 & 39.70 & 50.70 & 81.44 & 40.77 & 40.00 & 952.80 & 1216.80 & 1954.40 & 978.40 & 960.00 \\
\hline \multirow[b]{3}{*}{1 December } & 30 & 22.07 & 24.00 & 26.00 & 24.07 & 24.00 & 2.27 & 2.93 & $\mathbf{3 . 3 3}$ & 2.53 & 2.53 & 16.87 & 32.90 & 48.85 & 27.20 & 25.53 & 404.80 & 789.60 & 1172.80 & 652.80 & 612.80 \\
\hline & 60 & 24.00 & 24.07 & 24.53 & 24.13 & 24.00 & 2.60 & 3.23 & 3.43 & 2.63 & 2.57 & 33.53 & 50.63 & $\mathbf{7 3 . 3 3}$ & 34.73 & 35.53 & 804.80 & 1239.20 & 1760.00 & 833.60 & 852.80 \\
\hline & 90 & 24.20 & 24.07 & 25.33 & 22.07 & 22.13 & 2.63 & 3.07 & 3.27 & 2.50 & 2.43 & 27.83 & 42.03 & 63.77 & 30.73 & 29.46 & 668.00 & 1008.80 & 1530.40 & 737.60 & 707.20 \\
\hline \multicolumn{2}{|c|}{ LSD at 0.05} & & & 0.67 & & & & & 3.98 & & & & & 0.61 & & & & & 42.26 & & \\
\hline \multicolumn{22}{|c|}{$2008 / 2009$ season } \\
\hline \multirow[b]{3}{*}{15 October } & 30 & 22.00 & 23.27 & 24.00 & 24.00 & 22.00 & 2.20 & 2.77 & 3.12 & 2.37 & 2.47 & 10.98 & 30.20 & 38.66 & 12.30 & 19.00 & 263.20 & 724.80 & 928.00 & 295.20 & 456.00 \\
\hline & 60 & 22.20 & 24.20 & 24.53 & 22.20 & 22.00 & 2.50 & 3.17 & 3.50 & 2.70 & 2.67 & 18.65 & 36.53 & 57.31 & 27.60 & 25.07 & 447.20 & 876.80 & 1375.20 & 663.40 & 601.60 \\
\hline & 90 & 22.33 & 24.07 & 26.00 & 22.73 & 22.27 & 2.80 & 3.50 & 3.70 & 2.80 & 2.70 & 22.37 & 45.51 & 61.97 & 28.23 & 26.47 & 536.80 & 1092.00 & 1487.20 & 677.60 & 635.20 \\
\hline \multirow[b]{3}{*}{ 1November } & 30 & 22.40 & 24.40 & 24.33 & 22.53 & 22.47 & 2.63 & 3.07 & 3.27 & 2.73 & 2.67 & 27.17 & 35.93 & 60.43 & 29.00 & 25.00 & 652.01 & 862.40 & 1447.20 & 696.00 & 600.00 \\
\hline & 60 & 22.47 & 24.53 & 26.53 & 24.27 & 24.40 & 2.93 & 3.40 & 3.83 & 2.83 & 2.77 & 34.73 & 63.70 & 93.90 & 37.83 & 38.93 & 833.60 & 1528.80 & 2253.60 & 910.40 & 949.40 \\
\hline & 90 & 22.73 & 25.67 & 26.73 & 24.67 & 24.47 & 2.97 & $\mathbf{3 . 6 0}$ & 3.87 & $\mathbf{3 . 0 3}$ & 3.27 & 36.87 & 59.83 & 99.98 & 44.70 & 45.36 & 884.83 & 1436.00 & 2415.20 & 1072.8 & 1088.8 \\
\hline \multirow[b]{3}{*}{ 16November } & 30 & 22.27 & 24.00 & 26.40 & 22.27 & 22.13 & 2.50 & 3.13 & 3.40 & 2.53 & 2.47 & 18.20 & 35.57 & 70.40 & 26.05 & 23.33 & 318.00 & 853.60 & 1689.60 & 625.60 & 560.00 \\
\hline & 60 & 24.13 & 24.40 & 26.53 & 24.27 & 22.33 & 2.80 & 3.22 & 3.63 & 2.83 & 2.73 & 34.50 & 52.39 & 85.20 & 31.90 & 28.60 & 828.02 & 1257.60 & 2044.80 & 765.60 & 686.40 \\
\hline & 90 & 22.60 & 24.80 & 26.47 & 24.33 & 22.60 & 2.90 & 3.53 & 3.77 & $\mathbf{3 . 0 3}$ & 2.80 & 35.45 & 55.90 & 93.23 & 40.70 & 35.67 & 850.40 & 1341.60 & 2237.60 & 976.80 & 856.00 \\
\hline \multirow[b]{3}{*}{1 December } & 30 & 22.00 & 22.27 & 24.80 & 22.33 & 22.20 & 2.27 & 2.63 & 3.43 & 2.23 & 2.37 & 13.07 & 26.80 & 61.01 & 23.73 & 23.53 & 313.61 & 643.20 & 1464.80 & $\begin{array}{l}569.71 \\
\end{array}$ & 564.80 \\
\hline & 60 & 22.40 & 24.27 & 25.73 & 22.33 & 22.33 & 2.63 & 3.20 & 3.53 & 2.93 & 2.77 & 28.13 & 42.47 & $\begin{array}{l}67.87 \\
\end{array}$ & 28.97 & 29.70 & 675.21 & 1020.00 & 1628.80 & 695.20 & 712.80 \\
\hline & 90 & 22.47 & 24.13 & 25.53 & 22.20 & 22.07 & 2.80 & 3.20 & 3.50 & 2.87 & 2.73 & 30.07 & 41.43 & 68.13 & 34.37 & 31.57 & 721.61 & 994.40 & 1635.20 & 824.80 & 757.61 \\
\hline \multicolumn{2}{|c|}{ LSD at 0.05} & \multicolumn{5}{|c|}{0.38} & \multicolumn{5}{|c|}{0.17} & \multicolumn{5}{|c|}{4.27} & \multicolumn{5}{|c|}{113.04} \\
\hline
\end{tabular}


means that the performance of Serw-4variety under climatic conditions of Nov. $1^{\text {st }}$ sowing date was better than others. Also, its efficiency in uptake and utilization of nitrogen at all $\mathrm{N}$ levels was better than other. For that, it showed the highest values for seed yield per plant and its components at all $\mathrm{N}$ levels as well as all sowing dates compared to other varieties.

According to these results it could be concluded that to maximize canola productivity under the conditions of the Sadat city area, Serw-4 variety could be sown at early Nov. and $\mathrm{N}$ fertilization should be applied at $90 \mathrm{Kg} \mathrm{N} /$ fadden in three doses, i.e. $20 \%$ at seeding $40 \%$ at 30 days of sowing , and $40 \%$ atflowering stage.

\section{REFERENCES}

Amany M. R. (2009). Evaluation of certain canola varieties for tolerance to cabbage aphid under some agricultural practices. $1^{\text {st }}$ Nile Delta Conf.on Export Crops, Fac.of Agric., Minufiya Univ.(2009):227-244.

Asfour M.M.,(2006). Genetical and physiological studies on canola. Ph.D. Thesis, Fac. Agric., Minufiya Univ., Egypt.

Chauhan A . K., Singh M. and Dadhwal K . S. (1993). Effect of nitrogen level and row spacing on the performance of rape ( Brassica napus, L ) Indian J . Agron. 37 (4) : 851-853.

Cottenie L., Verloo M., Kiekens L., Velghe G. and Camerlyck R. (1982). Chemical analysis of plants and soils in laboratory of analytical and geochemistry . State Univ . Ghernt, Belguium , pp. 33-111.

Daneshian A.M., Ahmadzadeh A.R., Shahriar H.A.and Khanizadeh A. R. (2008). Effect of sowing dates on grain and biological yield, oil and meal protein percentage in three cultivars of rape (Brassica napus L.,) Rese. J .of Bio . Scie 3 (7): 729 - 732. Medwell . J. online 2008.

El- Tantawy N. and. Soliman S . Z ( 1999 ). The impact of reducing cultivated areas of some important oil crops on the Egyptian oil production . J . Agric . Sci . Mansoura Univ. $24: 7459-7469$.

Fathi, G., Sidadt S. A. and Hemaiaty S.S. (2005). Effect of sowing dates on yield and yield components of three oilseed rape varieties . Acta. Agron.Hung., 51 (3) :249-255 .

Francois L. E. (1994). Growth seed yield and oil content of canola grown under saline conditions .Agron.J.86: 233-237 .

Gomez K. A. and Gomez A.A. (1984 ) Statistical procedures for Agric.1 Res.. $2^{\text {nd }}$ Ed John Wiley and Sons, Inc .

Gul.H . and Ahmad R.. (2007): Effect of different sowing dates on the vegetative and reproductive growth of canola ( Brassica napus L.) cultivars under different salinity. Pak. J. Bot., 39 (4) :1161-1172.

Hassan K . H . and El - Hakeem M . S . (1996). Response of some rape seed cultivars to nitrogen rates and plant density under saline condition at Siwa Oasis . Ann. of Agric. Sci. Cairo, 41 (1 ) : 229 - 242.

Jasinska Z., Koteck A., Malarz W., Horodyski A., Musnicka B., Musnicki C., Jodlowski M., Budzynski W., Majkowski K., Wrobel E. and Sikora B, ( 1989 ) . Effect of sowing dates and sowing rate on the development and seed yield of winter rape cultivars . Buile . Institi . Hodowli - I - Aklimatyzacji Roslin . 169 ; 111-119.

Jenkins P. D. and Leitch M . H . (1986). Effect of sowing dates on the growth and yield of winter oil seed rape (Brassica napus, L.) . J. of Agric . Sci . UK. 107 ( 2 ) ; 405 - 420.

Leilah A. A, Al- Khateeb S. A., Al- Thabet S.S. and Al-Barrak K . M..(2003). Influence of sowing dates and nitrogen fertilizer on growth and yield of canola. Zagazig . J . Agric . Res ., Vol . 30 No . ( 3 ) 2003 . 591605.

Leto C., Carrubba A ., Cibella R. and Trapani P. (1995). Effect of sowing dates and cultivar on phenology and yield of autumn sown oil seed rape Brassica napus, L., Var Oleifera). Rivista de Agronomia , 29 (1): 72 $-82$.

Miralles D. J., Ferro B. C. and Slafer G. A. (2001) .Developmental responses to sowing dates in wheat, barley and rape seed . Field Crops Research, 71, Inssue: 3, 20 July 2001, 211-223.

Mohsen B., Mahmod D. and Ahmad G. P.. (2004). The effect of sowing dates and seeding rate on yield and yield components of rape seed on dry land. Golestan . Agric. and Nat. Resources Res. Gorgan, Iran . Agris Record No . IR2006000631 (www. Agrisis . org ).

Ozer H. (2002). Sowing dates and nitrogen rate effects on growth, yield and yield components of summer rapeseed cultivars. Eup . J . Agron., 19 ( 3 ) : 453-463.

Patel J. R. ( 1998 ). Effects of irrigation and nitrogen on mustard . J. Maharashtra Agric., Univ. Publ. 23; 259-261. (CAB Abstracts).

Said E. M. and M. M. Keshta .(1999 ). Response 
of some canola (Brassica napus L.) cultivars to different nitrogen fertilization levels . J . Agric . Sci . Mansoura Univ. , 24 (4): $1689-1697$.

Saini K. S. and Sidhu J . S. (1997). Effect of different levels of nitrogen and row spacing on the yield performance of gobhi sarson (Brassica napus, L ) sown on different dates. Ann. Of Agic . Bio .Res., 2 (2) : 125127.

Sarandon S.J., Chamorro A., Bezus R. and Gianibelli M.C.(1993). Response to nitrogen fertilizer of rape ( Brassica napus var oliefra ).Effect of biomass production , seed yield and its components. Revista de la Facultat de Agronmia La plata 69 1:63-67 (C. F Soil \&Fertilizer Abst., 58 1, 9257, (1995).

Shah.S.A and Rahman K. (2009). Yield and growth response of rapeseed (Brassica napus L.,) mutants to different seeding rates and sowing dates. Pak. J. Bot., 41 (6): 27112716.

Sharief.A.E.and Keshta M. M. (2000). Response of some canola cultivars (Brassica napus, L.) to different sources and levels of nitrogen fertilizer in soil affected by salinity. Zagazig J . Agric . REs., 27 : 603616.

Sharief A.E. and Keshta M. M. (2002). Influence of sowing dates and plant density on growth and yield of canola ( Brassica napus , L.,) under salt affected soils in Egypt . Scie . J . of King Faisal Uni (Basic and Applied Sciences) . Vo. 3 No.1. 65-75.

Sing V. (1984). Research and development strategies for oil seed production in India (Ed),ICAR,New Delhi,pp. 11-44.

Singh B . N .and Srivastava S . P. ( 1986 ) Effect of irrigation and nitrogen fertilization on growth and yield of mustard in mid hills of Meghalaya. Indian Agron .31: 135- 138.

Singh T. P. and Singh H . P. (1984). Dry matter accumulation and nutrient uptake in Indian rape as affected by plant density and fertilizers . Indian J . Agron . 29 : 522-527.

Starner D. E., Bhardwaj L., Hamama A.A., Muddappa R., Rangappa M.and Janick J. (1996). Canola production in Virginia. The third National Symposium. Indianapolis, 22-25 Oct. Indiana (C.F.CD Rom Computer system).

Taylor A J. and Smith C . J., (1992). Effect of sowing dates and seeding rate on yield and yield components of irrigated canola (Brassica napus L.) grown on red- brown earth in south eastern Australia. Aus. J. Agric . Res ., 43 (7): 1629-1641.

Thomas D.L., Breve M.A., Raymer P.L., Dasilva J.F.K.and K.Silva J.F. (1990). Planting dates effect and double-cropping potential of rain the southeastern United States . Applied Agric. Res.,5(3):205-211.

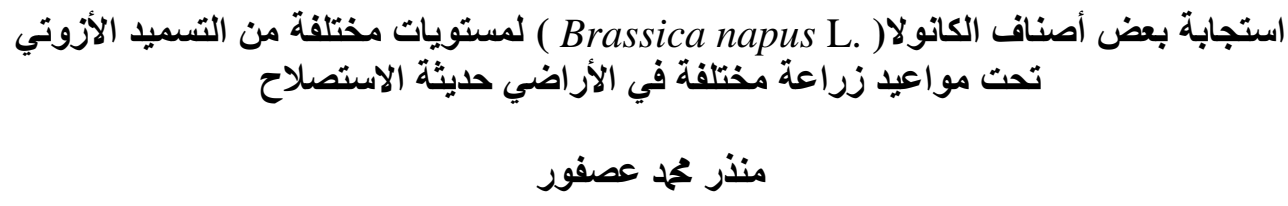

معهد الاراسات والبحوث البيئيةـ- جامعة المنوفية ـفرع السادات

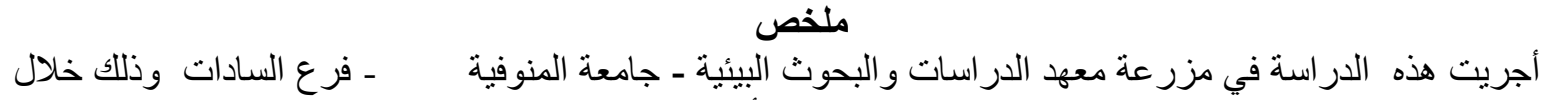

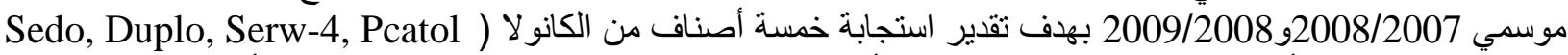

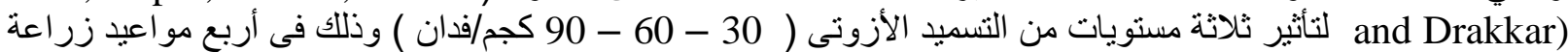

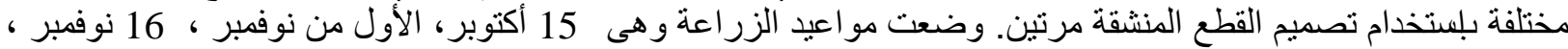

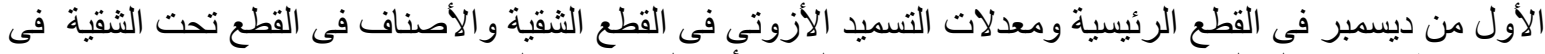

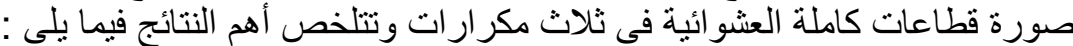
1- أفضل ميعاد زر اعة هو الأول من نوفمبر حيث أعظة أعطى أعلى القيم فى معظم الصفات تحت الدر استة مسجلا أعلى محصول بذور للفدان( 1050.13,1175.40 كجم ) كذا أعلى محصول بذورللنبات ( التو الى . بلى 
2- كان أفضل مستوى من التسميد الأزوتى 90كجم وحدة أزوت للفدان حيث أعطى أعلى القيم فى معظم الصفات تحت

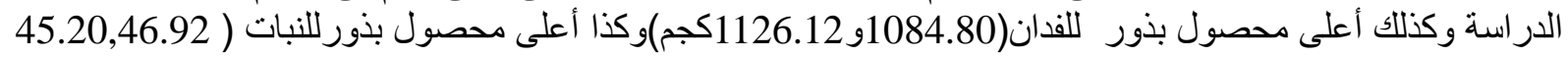

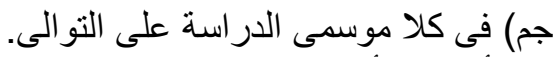

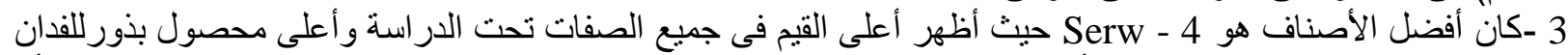

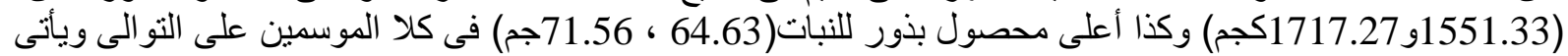

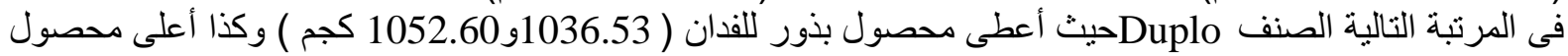

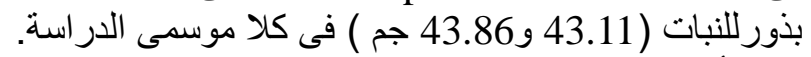

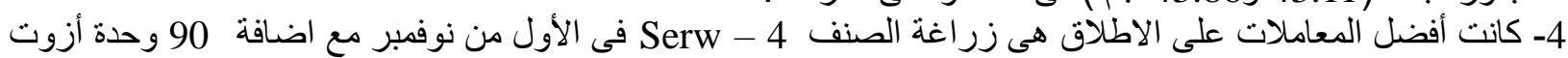

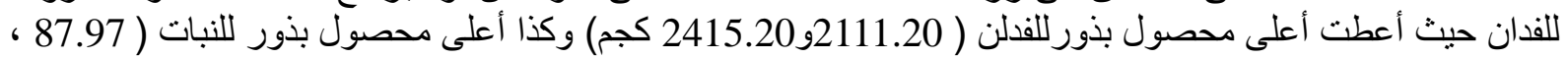

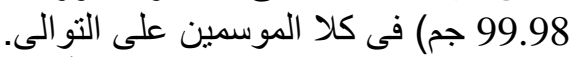

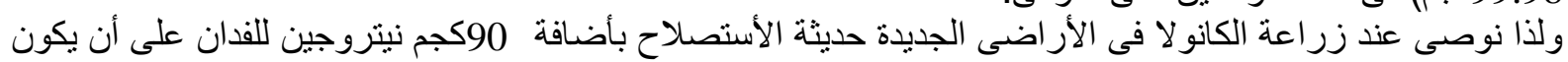

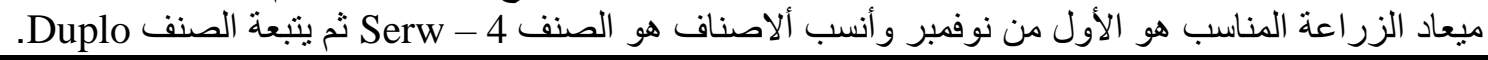

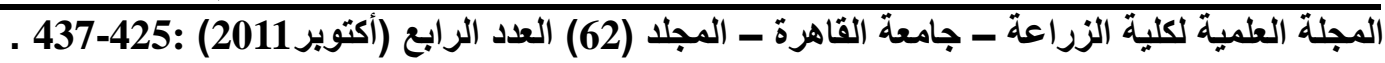

